\title{
On switching classes of graphs
}

\author{
B. Et-Taoui and A. Fruchard
}

June 1, 2015

\begin{abstract}
A class of non-oriented simple graphs is called Seidel switching self-complementary if the complement of any representing graph is in the same equivalence class. This note introduces the 3 -signature $(s, t)$ of a switching class of $n$-vertex graphs. The numbers $s$ and $t$ are the numbers of positive and negative triples within any representing graph of the class. It appears that, for any switching self-complementary class of $n$-vertex graphs, these numbers are equal, yielding $\left(\begin{array}{l}n \\ 3\end{array}\right)$ even. Consequently if $n \equiv 3(\bmod 4)$ then there is no switching self-complementary class of $n$-vertex graphs. We then consider the switching classes of Paley conference graphs with $4 k+2$ vertices, $4 k+1=p^{\alpha}, p$ an odd prime and $\alpha$ a positive integer. We reprove that these classes are switching selfcomplementary. Moreover, it is proven that all $4 k$-vertex graphs contained in a $(4 k+2)$ vertex Paley conference graph are switching equivalent and their class is still a switching self-complementary class. In addition, the 3 -signature is generalized in view of obtaining a complete invariant of switching classes up to order 8 .
\end{abstract}

Keywords: Seidel switching, self-complementary, signature, Paley conference graph. AMS Classification: 05C60, 05C50, 51A05.

\section{Introduction}

We deal with simple non-oriented graphs. Two graphs are called isomorphic if there is a oneto-one correspondence between their vertex-sets which preserves adjacency. The complement $\bar{G}$ of a graph $G$ has the same vertex-set as $G$ but two vertices of $\bar{G}$ are adjacent in $\bar{G}$ if and only if they are not adjacent in $G$. A graph is self-complementary if it is isomorphic to its complement. There is a huge literature about self-complementary graphs, see e.g. $[4,5,13,14]$ and the references therein.

In this article, we are concerned with another notion of self-complementarity. Given a graph, a Seidel switching at any vertex consists of erasing the existing edges and adding the nonexisting ones. Two graphs are called Seidel switching equivalent if one can pass from one graph to the other by Seidel switchings. A Seidel switching self-complementary class of graphs (s.s.c. for short) is a class of graphs in which any representing graph is Seidel switching equivalent to its complement. This notion has been introduced by van Lint and Seidel [9] in relation with equiangular lines of the Euclidean space, see Section 2. This geometric setting yields quickly our first result: With the use of the shape invariant of triples $[1,2]$ we obtain that a necessary condition for a class of $n$-vertex graphs to be s.s.c. is that $\left(\begin{array}{l}n \\ 3\end{array}\right)$ be even. This result already appeared in [7], but with different methods.

In 1933, Paley [11] used the Legendre symbol on finite fields to construct symmetric conference matrices of order $p^{\alpha}+1, p$ prime, $p^{\alpha} \equiv 1(\bmod 4)$. We call the corresponding graph 
a Paley conference graph. We recall this construction in Section 3. In [14], Sachs proved that the well-known $p^{\alpha}$-vertex Paley graph is self-complementary; in [6] the authors proved that all $p^{\alpha}$-vertex subgraphs of the Paley conference graph are switching equivalent, hence they are representing graphs of a s.s.c. class. In this article we pursue this road one step more, proving that all $\left(p^{\alpha}-1\right)$-vertex subgraphs of a Paley conference graph are also switching equivalent and that their class is s.s.c.

In Section 4 we introduce a more general notion of signature of a switching class of graphs and we prove that this signature with the determinant form a complete invariant up to order 8 at least for the number of vertices. The question whether this remains true for all orders is analogous in our context of the well-known Kelly-Ulam reconstruction conjecture [8, 12]. Another related question of reconstruction in the context of vertex-switching was asked by Stanley [16]. Note that these three questions are independent.

\section{Equiangular lines and Seidel switching classes of graphs}

A $n$-tuple of lines of $\mathbb{R}^{d}$ is called equiangular of parameter $\left.a \in\right] 0,1[$ if, for any two lines and any unit vectors $x, y$ on each of them, the inner product of $x$ and $y$ satisfies $\langle x, y\rangle \in\{-a, a\}$, i.e. the $n$-tuple is a tuple of equidistant points in the elliptic space with the distance $d([x],[y])=$ $\arccos a$.

It is known [9] that there is a one-to-one correspondence between congruence classes of equiangular dependent $n$-tuples of lines and Seidel switching equivalence classes of $n$-vertex graphs. We present this correspondence below.

Let $\left(l_{1}, \ldots, l_{n}\right)$ be an equiangular dependent $n$-tuple of lines of $\mathbb{R}^{d}$ of parameter $\left.a \in\right] 0,1[$. Choose unit vectors $x_{i} \in l_{i}$ arbitrarily. Then the Gram matrix $\left(\left\langle x_{i}, x_{j}\right\rangle\right)_{1 \leq i, j \leq n}$ is of the form $I_{n}+a M$, where $M$ is symmetric, with diagonal entries all zero and other entries \pm 1 . We call such a matrix a Seidel matrix. To a Seidel matrix $M=\left(a_{i j}\right)_{1 \leq i, j \leq n}$ is associated the Seidel graph $G$ with vertex set $V=\left\{v_{1}, \ldots, v_{n}\right\}$ and with an edge between $v_{i}$ and $v_{j}$ if and only if $a_{i j}=-1$.

Changing the order of the lines in the $n$-tuple corresponds to changing the order of the rows and corresponding columns of the associated Seidel matrix $M$ and to changing the order on the vertices in the corresponding Seidel graph; changing the choice of unit vector $x_{i}$ in the line $l_{i}$ correponds to multiplying the $i$-th row and column of $M$ by -1 and to carrying out the Seidel switching at the $i$-th vertex of the graph described in our introduction. These two operations on Seidel matrices and Seidel graphs generate the so-called Seidel switching equivalence relation on matrices, resp. graphs.

Conversely, to each switching class of graphs with smallest eigenvalue $\lambda_{0}$, corresponds a congruence class of equiangular tuples of lines of parameter $a=-\frac{1}{\lambda_{0}}$.

It is known $[1,2]$ that a triple $([x],[y],[z])$ of $\mathbb{R P}^{d-1}$ is determined by its side lengths and its shape invariant $\tau([x],[y],[z])=\frac{\langle x, y\rangle\langle y, z\rangle\langle z, x\rangle}{|\langle x, y\rangle\langle y, z\rangle\langle z, x\rangle|}$. We call positive a triple such that $\tau([x],[y],[z])=$ 1.

The 3 -signature of a $n$-tuple in $\mathbb{R P}^{d-1}$ is the pair $(s, t)$ of integers, where $s$ denotes the number of positive triples in $X$ and $t$ is the number of negative triples in $X$. Thus we have $s+t=\left(\begin{array}{l}n \\ 3\end{array}\right)$. This 3 -signature is invariant by Seidel switching. Now observe that a positive triple in $\bar{G}$ corresponds to a negative one in $G$. As a consequence, if the 3-signature of $G$ is $(s, t)$ then the 3 -signature of $\bar{G}$ is $(t, s)$. This proves our first result.

Theorem 1. If a switching class of $n$-vertex graphs is a s.s.c. class then its 3 -signature is of the form $(s, s)$. As a consequence $\left(\begin{array}{l}n \\ 3\end{array}\right)$ is necessarily even. In other words there is no s.s.c. class 
of $n$-vertex graphs if $n \equiv 3(\bmod 4)$.

Remarks. 1. van Lint and Seidel [9] already observed that there is no s.s.c. class of 7-vertex graphs by looking at the spectrum of the adjacency matrix of a representing graph for each of the 54 switching equivalence classes. Theorem 1 gives a simple geometric expanation of this observation.

2. Greaves, Koolen, Munemasa and Szöllösi [7] obtained the same result (Proposition 3.7) using different methods.

3. The 3-signature is a weaker invariant than the spectrum, as shows the following statement.

Proposition 2. (a) If two switching classes of graphs have the same spectrum, then they have the same 3-signature.

(b) If a switching class of graphs has a spectrum symmetric with respect to 0, then its 3signature is of the form $(s, s)$.

(c) There exist noncospectral graphs with the same 3-signature.

Proof. (a) Let $G$ and $G^{\prime}$ be two $n$-vertex representing graphs of two distinct classes with the same 3-signature $(s, t)$, resp. $\left(s^{\prime}, t^{\prime}\right)$. Let $M$ and $M^{\prime}$ be their associated Seidel matrices and let $P_{M}(X)=\operatorname{det}\left(X I_{n}-M\right)=\sum_{k=0}^{n} a_{k} X^{k}$ and $P_{M^{\prime}}(X)=\operatorname{det}\left(X I_{n}-M^{\prime}\right)=\sum_{k=0}^{n} a_{k}^{\prime} X^{k}$ be the corresponding characteristic polynomials. If $G$ and $G^{\prime}$ are cospectral, then $a_{k}=a_{k}^{\prime}$ for all $k=0, \ldots, n$. It is known that, for every $k \in\{0, \ldots, n-1\}$ the coefficient $a_{k}$ is equal to the sum of all $\left(\begin{array}{l}n \\ k\end{array}\right)$ principal minors of order $n-k$ of $M$. In particular for $k=n-3$, the principal minors of order 3 have two values: 2 if the corresponding triple is positive and -2 if it is negative, yielding $2(s-t)=a_{n-3}=a_{n-3}^{\prime}=2\left(s^{\prime}-t^{\prime}\right)$. Since $s+t=s^{\prime}+t^{\prime}=\left(\begin{array}{l}n \\ 3\end{array}\right)$, we obtain $s=s^{\prime}$ and $t=t^{\prime}$

(b) If $G$, of 3 -signature $(s, t)$, has a spectrum symmetric with respect to 0 , then it is cospectral to its complement, whose 3 -signature is $(t, s)$, hence $s=t$ from item (a).

(c) The four s.s.c. classes of 6-vertex graphs (see the middle of the last line of Table 4.1 of [9]) have 3 -signature $(10,10)$ and have distinct spectra.

We will proceed a generalization of this 3-signature in Section 4.

\section{Paley conference s.s.c. classes of graphs}

For completeness, we recall below the contruction of Paley [11] of symmetric conference matrices. We present the construction of Geothals and Seidel [6].

Let $p$ be an odd prime, $\alpha$ a positive integer and put $q=p^{\alpha} \equiv 1(\bmod 4)$. Let $F_{q}=$ $\left\{a_{1}, \ldots, a_{q}\right\}$ denote any $q$-element field (all are isomorphic) with $a_{1}=0$ and set $F_{q}^{*}=F_{q} \backslash\{0\}$. Let $\varkappa: F_{q} \rightarrow\{-1,0,1\}$ denote the Legendre symbol, defined by $\varkappa(0)=0, \varkappa(a)=1$ if $a \neq 0$ is a square in $F_{q}$ and $\varkappa(a)=-1$ otherwise. We recall that $\varkappa$ is a character, i.e. $\varkappa(a) \varkappa(b)=\varkappa(a b)$, and that $\sum_{a \in F_{q}} \varkappa(a)=0$.

Let $V$ be a two-dimensional vector space over $F_{q}$, let det be any nonzero alternating bilinear form on $V$, and let $x, y \in V$ be independant. Put $y_{0}=x$ and, for $1 \leq k \leq q, y_{k}=y+a_{k} x$. The Paley matrix is the $(q+1) \times(q+1)$ matrix $C=\left(c_{k l}\right)_{0 \leq k, l \leq q}$ whose entry on the $k$-th row and $l$-th column, $0 \leq k, l \leq q$, is $c_{k l}=\varkappa\left(\operatorname{det}\left(y_{k}, y_{l}\right)\right)$. One finds

$$
C=\varkappa(\operatorname{det}(x, y))\left(\begin{array}{cc}
0 & j \\
j^{T} & \left(\varkappa\left(a_{k}-a_{l}\right)\right)_{1 \leq k, l \leq q}
\end{array}\right),
$$


where $j$ is the all-one line vector and $j^{T}$ its transpose.

One can see that $C$ is a real symmetric conference matrix, i.e. $C^{2}=q I_{q+1}$, using the Jacobsthal formula: if $b \in F_{q}^{*}$, then $\sum_{a \in F_{q}} \varkappa(a) \varkappa(a+b)=-1$.

Theorem 3. The switching equivalence class of $(q+1)$-vertex Paley conference graphs is a s.s.c. class. Moreover the descendants of any Paley conference graph are switching equivalent, the descendants of the descendants, that is the $(q-1)$-vertex graphs, are also switching equivalent and the two classes are s.s.c. classes.

Remarks. 1. Since $q \equiv 1(\bmod 4)$, by Theorem 1 , the switching class of the corresponding conference Paley graph cannot contain any s.s.c. class of $(q-2)$-vertex graphs.

2. The first item of Theorem 3 was quoted without proof in [3]. The second item is proven in $[6,14]$. The third item is new up to our knowledge.

Proof. We prove that $G$ is a representing graph of a s.s.c. class. Choose a non-square element $\gamma \in F_{q}$ and consider the vectors $\widetilde{y}_{k}$ given by $\widetilde{y}_{0}=\gamma x$ and, for $1 \leq k \leq q, \widetilde{y}_{k}=y+a_{k} \gamma x$. Then we have $\operatorname{det}\left(\widetilde{y}_{k}, \widetilde{y}_{l}\right)=\gamma \operatorname{det}\left(y_{k}, y_{l}\right)$, hence the matrix $\widetilde{C}=\left(\varkappa\left(\operatorname{det}\left(\widetilde{y}_{k}, \widetilde{y}_{l}\right)\right)\right)_{0 \leq k, l \leq q}$ is equal to $-C$, i.e. is associated to $\bar{G}$. Let $\sigma \in S_{q}$ be the permutation such that $\gamma a_{k}=a_{\sigma(k)}$ for all $k=1, \ldots, q$. We multiply the first row and the first column of $C$ by -1 and we reorder the lines and columns from 2 to $q+1$ according to $\sigma$ to recover $-C$.

We then prove that the $q$-vertex graph $\widetilde{G}$ obtained by deleting the first vertex is in a s.s.c. class. The corresponding Seidel matrix is $P=\varkappa(\operatorname{det}(x, y))\left(\varkappa\left(a_{k}-a_{l}\right)\right)_{1 \leq k, l \leq q}$. The same permutation $\sigma$ such that $\gamma a_{k}=a_{\sigma(k)}$ on the rows and columns transforms $P$ to $P^{\sigma}=$ $\varkappa(\operatorname{det}(x, y))\left(\varkappa\left(a_{\sigma(k)}-a_{\sigma(l)}\right)\right)_{1 \leq k, l \leq q}=\varkappa(\operatorname{det}(x, y))\left(\varkappa(\gamma) \varkappa\left(a_{k}-a_{l}\right)\right)_{1 \leq k, l \leq q}=-P$ since $\varkappa(\gamma)=$ -1 . Observe that we did not need to multiply any row/column by -1 , i.e. $\widetilde{G}$ is selfcomplementary in the usual sense. This was first proved by Sachs [14] and his complementing permutation is the same as ours.

We now delete also the second vertex. Since we chose $a_{1}=0$, the matrix

$$
Q=\varkappa(\operatorname{det}(x, y))\left(\varkappa\left(a_{k}-a_{l}\right)\right)_{2 \leq k, l \leq q}
$$

is still equivalent to $-Q$ with the same permutation $\sigma$.

We already proved that $G, G \backslash\left\{v_{1}\right\}$, and $G \backslash\left\{v_{1}, v_{2}\right\}$ belong to s.s.c. classes. It remains to prove that all descendants, resp. descendants of descendants, of $G$ are switching equivalent.

We begin with the descendants. Let $k_{1} \in\{1, \ldots, q\}$ arbitrary and set $\widetilde{y}_{0}=\widetilde{x}=y_{k_{1}}$ and, for $k \geq 1, \widetilde{y}_{k}=y+a_{k} \widetilde{x}$ (with the same $a_{k}$ as for $\left.y_{k}\right)$. Then we have $\widetilde{y}_{k}=\left(1+a_{k}\right) y+a_{k} a_{k_{1}} x$ for all $k \geq 1$. If $a_{k}=-1$, then $\widetilde{y}_{k}=-a_{k_{1}} x=-a_{k_{1}} y_{0}$, otherwise $\widetilde{y}_{k}=\left(1+a_{k}\right)\left(y+\frac{a_{k} a_{k_{1}}}{1+a_{k}} x\right)$.

Put $\gamma_{k}=-a_{k_{1}}$ if $a_{k}=-1, \gamma_{k}=1+a_{k}$ otherwise. Choose the permutation $\sigma \in S_{q}$ such that $a_{\sigma(k)}=0$ if $a_{k}=-1$, and $a_{\sigma(k)}=\frac{a_{k} a_{k_{1}}}{1+a_{k}}$ otherwise. In this manner, we have $\widetilde{y}_{k}=\gamma_{k} y_{\sigma(k)}$, hence $\varkappa\left(\operatorname{det}\left(\widetilde{y}_{k}, \widetilde{y}_{l}\right)\right)=\varepsilon_{k, l} \varkappa\left(\operatorname{det}\left(y_{\sigma(k)}, y_{\sigma(l)}\right)\right)$ with $\varepsilon_{k, l}=\varkappa\left(\gamma_{k}\right) \varkappa\left(\gamma_{l}\right)$.

For each $k$ such that $\gamma_{k}$ is not a square, we multiply the $k$-th row and the $k$-th column by -1 . This sequence of switchings, combined with the permutation $\sigma$, transforms $\widetilde{C}=\left(\varkappa\left(\operatorname{det}\left(\widetilde{y}_{k}, \widetilde{y}_{l}\right)\right)\right)_{0 \leq k, l \leq q}$ into $C$.

The proof that the descendants of descendants of $G$ are switching equivalent is very similar. Let $k_{1}, k_{2} \in\{1, \ldots, q\}$ arbitrary with $k_{1} \neq k_{2}$; the case $k_{1}$ or $k_{2}=0$ will be treated later on. Set $\widetilde{x}=y_{k_{1}}, \widetilde{y}=y_{k_{2}}, \widetilde{y}_{0}=\widetilde{x}$, and, for $k \geq 1, \widetilde{y}_{k}=\widetilde{y}+a_{k} \widetilde{x}$. Then we have $\widetilde{y}_{0}=\widetilde{x}$ and, for $k \geq 1, \widetilde{y}_{k}=\left(1+a_{k}\right) y+\left(a_{k_{2}}+a_{k} a_{k_{1}}\right) x$. If $a_{k}=-1$, then $\widetilde{y}_{k}=\left(a_{k_{2}}-a_{k_{1}}\right) y_{0}$, otherwise $\widetilde{y}_{k}=\left(1+a_{k}\right)\left(y+\frac{a_{k_{2}}+a_{k} a_{k_{1}}}{1+a_{k}} x\right)$. 
Put $\gamma_{k}=a_{k_{2}}-a_{k_{1}}$ if $a_{k}=-1, \gamma_{k}=1+a_{k}$ otherwise, and choose the permutation $\sigma \in S_{q}$ such that $a_{\sigma(k)}=0$ if $a_{k}=-1$, and $a_{\sigma(k)}=\frac{a_{k_{2}}+a_{k} a_{k_{1}}}{1+a_{k}}$ otherwise. Then we have $\widetilde{y}_{k}=\gamma_{k} y_{\sigma(k)}$, hence $\varkappa\left(\operatorname{det}\left(\widetilde{y}_{k}, \widetilde{y}_{l}\right)\right)=\varepsilon_{k, l} \varkappa\left(\operatorname{det}\left(y_{\sigma(k)}, y_{\sigma(l)}\right)\right)$ with $\varepsilon_{k, l}=\varkappa\left(\gamma_{k}\right) \varkappa\left(\gamma_{l}\right)$.

For each $k$ such that $\gamma_{k}$ is not a square, we multiply the $k$-th row and the $k$-th column by -1 . This sequence of switchings, combined with the permutation $\sigma$, transforms $\widetilde{C}=\left(\varkappa\left(\operatorname{det}\left(\widetilde{y}_{k}, \widetilde{y}_{l}\right)\right)\right)_{0 \leq k, l \leq q}$ into $C$.

In the case $k_{1}$ or $k_{2}=0$, say $k_{1}=0$, we set $\widetilde{x}=x, \widetilde{y}=y_{k_{2}}, \widetilde{y}_{0}=\widetilde{x}$, and, for $k \geq 1$, $\widetilde{y}_{k}=\widetilde{y}+a_{k} \widetilde{x}=y+\left(a_{k_{2}}+a_{k}\right) x$, hence we do not need $\gamma_{k}$ and we choose $\sigma \in S_{q}$ such that $a_{\sigma(k)}=a_{k}+a_{k_{2}}$.

\section{The $k$-signature}

It is known that the spectrum of a Seidel matrix does not characterize its Seidel switching class. We will give below the complete list of 8-vertex graphs which have the same spectrum (we say cospectral graphs) but are not in the same switching class. As a consequence, by Proposition 2 (a), the 3-signature of a graph cannot be a complete invariant. The purpose of this section is to generalize this 3-signature.

Given any integer $k \geq 3$, let $C_{1}, \ldots, C_{N_{k}}$ denote all Seidel switching classes of $k$-vertex graphs. Their total number $N_{k}$ is known to be also the number of Eulerian $k$-vertex graphs [10]. Given a $n$-vertex graph $G$ and $k \in\{3, \ldots, n-1\}$, we call $k$-signature of $G$ the $N_{k}$-tuple of integers $\left(\nu_{1}, \ldots, \nu_{N_{k}}\right)$ where $\nu_{j}$ is the number of $k$-vertex subgraphs of $G$ which belong to the class $C_{j}$. Since a sequence of switchings and a permutation on $G$ induce a sequence of switchings and a permutation on its subgraphs, this $k$-signature is an invariant by Seidel switching. Besides, we have $\nu_{1}+\cdots+\nu_{N_{k}}=\left(\begin{array}{l}n \\ k\end{array}\right)$. We call signature of a $n$-vertex graph $G$ the collection of all its $k$-signatures, $3 \leq k \leq n-1$. In the context of the classical equivalence of graphs, our $(n-1)$-signature corresponds to the notion of deck. Item (a) below is known as Kelly's lemma. We give a proof for completeness.

Proposition 4. (a) The $(n-1)$ signature of a $n$-vertex graph determines its whole signature. (b) The signature and the determinant of a graph determine its spectrum.

Proof. (a) Let $G$ be a $n$-vertex graph with vertex set $\left\{v_{1}, \ldots, v_{n}\right\}$. For each $i=1, \ldots, n$, let $G_{i}$ denote the $(n-1)$-vertex subgraph of $G$ with vertex set $\left\{v_{1}, \ldots, v_{i-1}, v_{i+1}, \ldots, v_{n}\right\}$, i.e. the graph obtained by erasing the $i$-th vertex $v_{i}$ and all edges adjacent to $v_{i}$. By assumption the switching class of $G_{i}$ is known, hence its whole signature. Fix $3 \leq k \leq n-2$ and let $S_{k, i}$ denote the $k$-signature of $G_{i}$. Then the $N_{k}$-tuple $\sum_{i=1}^{n} S_{k, i}$ gives the sum of all $k$-subgraphs of all $G_{i}$ in each switching class of $k$-vertex graphs. Notice that any $k$-vertex subgraph $H$ of $G$ is contained in exactly $n-k$ of the $G_{i}$, namely the $G_{i}$ such that $v_{i}$ is not a vertex of $H$. It follows that the $k$-signature of $G$ is $\frac{1}{n-k} \sum_{i=1}^{n} S_{k, i}$.

(b) Let $G$ be a $n$-vertex graph, $M$ its associated Seidel matrix, and $P_{M}(X)=\operatorname{det}(X I-M)=$ $X^{n}+\sum_{k=0}^{n-1} a_{k} X^{k}$ its characteristic polynomial. As already said, $a_{k}$ is the sum of all principal minors of order $n-k$ of $M$. We have $a_{n-1}=\operatorname{tr} M=0$ and $a_{n-2}=-\left(\begin{array}{l}n \\ 2\end{array}\right)$ since any principal minor of order 2 of $M$ is of the form $\left|\begin{array}{ll}0 & 1 \\ 1 & 0\end{array}\right|$ or $\left|\begin{array}{cc}0 & -1 \\ -1 & 0\end{array}\right|$, hence equal to -1 .

For each $k \in\{1, \ldots, n-3\}$, each Seidel switching class $C_{j}$ of $(n-k)$-vertex graphs and for any associated $(n-k) \times(n-k)$ Seidel matrix $M_{j}$, let $d_{j}=\operatorname{det} M_{j}$. If $\left(\nu_{1}, \ldots, \nu_{N_{n-k}}\right)$ is the $(n-k)$-signature of $G$, we then obtain $a_{k}=\nu_{1} d_{1}+\ldots \nu_{N_{n-k}} d_{N_{n-k}}$. 
Open questions 5. (a) Prove or disprove the following assertion: If two graphs have the same signature and the same determinant, then they are switching equivalent.

(b) Prove or disprove: If a graph has a symmetric signature, then it is in a s.s.c. class.

(c) Prove or disprove: If two n-vertex graphs have the same spectrum, and all their corresponding $(n-1)$-subgraphs have same spectra as well, then they are switching equivalent.

(d) For each integer $n$, determine the number of s.s.c. classes of $n$-vertex graphs.

The study below shows that Questions 5 (a) and (c) have an affirmative answer at least up to order 8. In view of Proposition 4, we only have to separate cospectral graphs. We used the list of switching classes of 8-vertex graphs available on the homepage of Ted Spence [15] and we found seven families of nonisomorphic cospectral graphs: one triple $\left(A_{1}, \bar{A}_{1}, A_{2}\right)$ with $A_{2}$ s.s.c., three pairs, denoted $\left(B_{1}, B_{2}\right),\left(C_{1}, C_{2}\right)$, and $\left(D_{1}, D_{2}\right)$, and the complementary pairs $\left(\bar{B}_{1}, \bar{B}_{2}\right)$, $\left(\bar{C}_{1}, \bar{C}_{2}\right)$, and $\left(\bar{D}_{1}, \bar{D}_{2}\right)$. Then, for each $8 \times 8$-matrix, we computed the spectrum of each $7 \times 7$ principal minor and we compared it to the list of spectra computed by van Lint and Seidel in [9]. At last, we observed that already the 7-signature separates the cospectral classes.

For convenience, instead of writing in extenso the 54-tuple forming the 7 -signature, we indicate the number of 7 -vertex subgraphs in each class only if that class contains such a subgraph, i.e. we do not indicate the zeroes. The reference [9] indicates only 27 of the 54 classes; the 27 missing are simply their complementaries, with the opposite spectrum; we indicate them with an overline. For instance, the abbreviated 7 -signature of the first class $A_{1}$, which is $\left(\overline{16}_{3}, 19_{3}, \overline{25}_{2}\right)$, means that, among its eight 7-vertex subgraphs, three are of type $\overline{16}$, the complementary of the graph numbered 16 in Table 4.2 of [9], three of type 19, and two of type $\overline{25}$. Observe that only $A_{2}$ has a symmetric 7 -signature; it is the only graph in a s.s.c. class.

Below is the complete list of switching nonequivalent cospectral 8-vertex graphs with their abbeviated 7-signature. For convenience, we provide a sketch of each graph $A_{1}$ to $D_{2}$. We found superfluous to draw also the complementary graphs $\bar{A}_{1}$ to $\bar{D}_{2}$. The numbering of the vertices refers to the order of the rows in [15]; the index refers to the class, as numbered in [9] Table 4.2, of the 7-vertex graph obtained when the vertex is removed. This class is indicated only for one vertex if several vertices lead to equivalent graphs.

Observe that, although $A_{1}$ and $\bar{A}_{1}$ have the same spectrum, the same automorphism group and the same number of non-isomorphic representing graphs of their classes [15], they are not equivalent since they have distinct 7 -signatures.
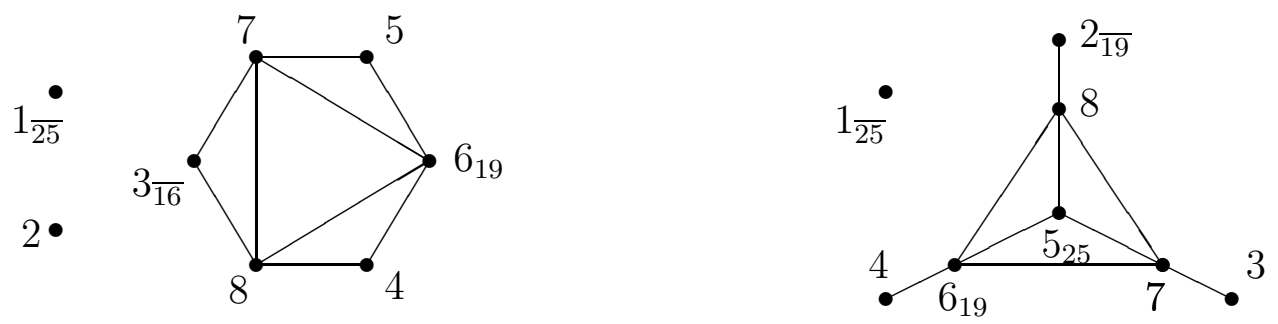

$$
\begin{aligned}
& A_{1} \rightsquigarrow\left(\overline{16}_{3}, 19_{3}, \overline{25}_{2}\right) \\
& \bar{A}_{1} \rightsquigarrow\left(16_{3}, \overline{19}_{3}, 25_{2}\right)
\end{aligned}
$$$$
A_{2} \sim \bar{A}_{2} \rightsquigarrow\left(19_{3}, \overline{19}_{3}, 25_{1}, \overline{25}_{1}\right)
$$

$$
P_{A_{i}}(x)=x^{8}-28 x^{6}+222 x^{4}-620 x^{2}+425
$$



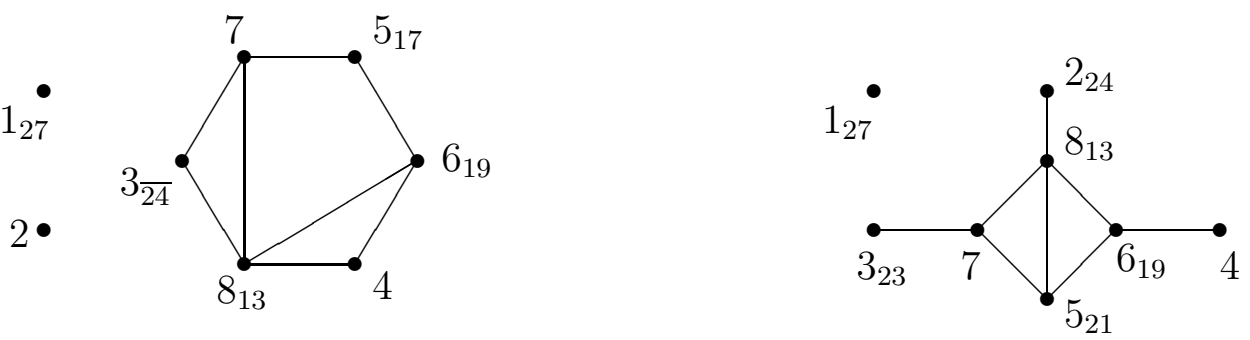

$$
\begin{gathered}
B_{1} \rightsquigarrow\left(13_{1}, 17_{1}, 19_{2}, \overline{24}_{2}, 27_{2}\right) \quad B_{2} \rightsquigarrow\left(13_{1}, 19_{2}, 21_{1}, 23_{2}, 24_{1}, 27_{1}\right) \\
P_{B_{i}}(x)=x^{8}-28 x^{6}-8 x^{5}+230 x^{4}+80 x^{3}-572 x^{2}-72 x+369
\end{gathered}
$$

$$
\begin{gathered}
\bar{B}_{1} \rightsquigarrow\left(\overline{13}_{1}, \overline{17}_{1}, \overline{19}_{2}, 24_{2}, \overline{27}_{2}\right) \quad \bar{B}_{2} \rightsquigarrow\left(\overline{13}_{1}, \overline{19}_{2}, \overline{21}_{1}, \overline{23}_{2}, \overline{24}_{1}, \overline{27}_{1}\right) \\
P_{\bar{B}_{i}}(x)=x^{8}-28 x^{6}+8 x^{5}+230 x^{4}-80 x^{3}-572 x^{2}+72 x+369
\end{gathered}
$$
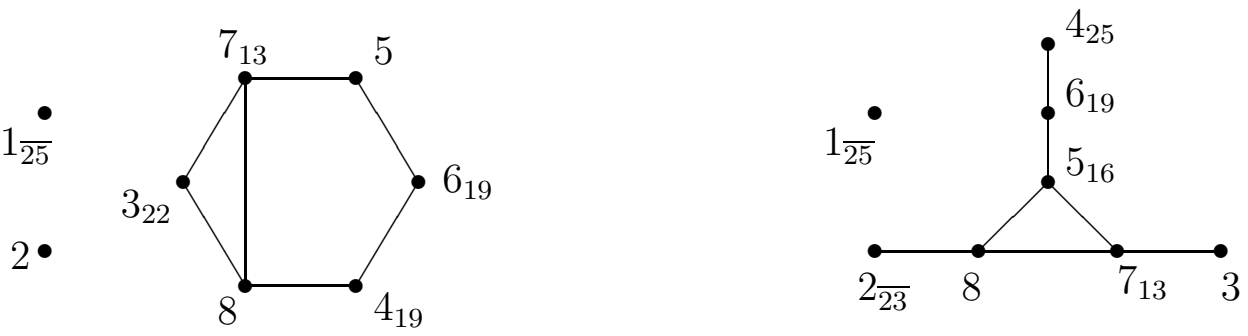

$$
\begin{gathered}
C_{1} \rightsquigarrow\left(13_{2}, 19_{3}, 22_{1}, \overline{25}_{2}\right) \quad C_{2} \rightsquigarrow\left(13_{2}, 16_{1}, 19_{1}, \overline{23}_{2}, 25_{1}, \overline{25}_{1}\right) \\
P_{C_{i}}(x)=x^{8}-28 x^{6}-8 x^{5}+230 x^{4}+80 x^{3}-636 x^{2}-200 x+305
\end{gathered}
$$

$$
\begin{gathered}
\bar{C}_{1} \rightsquigarrow\left(\overline{13}_{2}, \overline{19}_{3}, \overline{22}_{1}, 25_{2}\right) \quad \bar{C}_{2} \rightsquigarrow\left(\overline{13}_{2}, \overline{16}_{1}, \overline{19}_{1}, 23_{2}, 25_{1}, \overline{25}_{1}\right) \\
P_{\bar{C}_{i}}(x)=x^{8}-28 x^{6}+8 x^{5}+230 x^{4}-80 x^{3}-636 x^{2}+200 x+305
\end{gathered}
$$

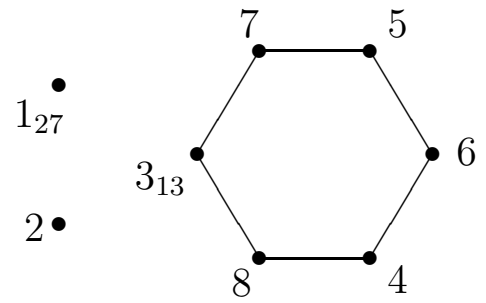

$$
D_{1} \rightsquigarrow\left(13_{6}, 27_{2}\right)
$$$$
P_{D_{i}}(x)=x^{8}-28 x^{6}-16 x^{5}+222 x^{4}+160 x^{3}-492 x^{2}-144 x+297
$$

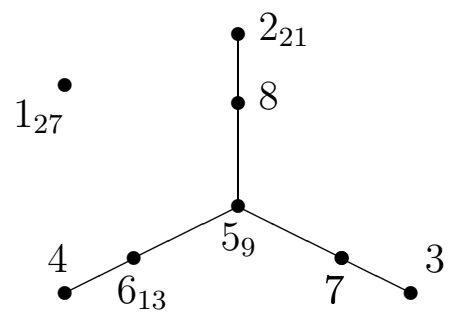

$D_{2} \rightsquigarrow\left(9_{1}, 13_{3}, 21_{3}, 27_{1}\right)$

$\bar{D}_{1} \rightsquigarrow\left(\overline{13}_{6}, \overline{27}_{2}\right) \quad \bar{D}_{2} \rightsquigarrow\left(\overline{9}_{1}, \overline{13}_{3}, \overline{21}_{3}, \overline{27}_{1}\right)$

$P_{\bar{D}_{i}}(x)=x^{8}-28 x^{6}+16 x^{5}+222 x^{4}-160 x^{3}-492 x^{2}+144 x+297$ 


\section{References}

[1] U. Brehm, The shape invariant of triangles and trigonometry in two-point homogeneous spaces, Geom. Dedicata 33 (1990) 59-76

[2] U. Brehm, B. Ettaoui, Congruence criteria for finite subspaces of complex projective and complex hyperbolic spaces, Manuscripta Math. 96 (1998) 81-95

[3] F. C. Bussemaker, R. A. Mathon, J. J. Seidel, Tables of two-graphs, Combinatorics and graph theory (Calcutta, 1980), Springer Lecture Notes Math. 885 (1981) 70-112

[4] A. Farrugia, Self-complementary graphs and generalisations: a comprehensive reference manual, Thesis, University of Malta (1999)

[5] R. A. Gibbs, Self-Complementary Graphs, J. Combinatorial Theory (B) 16 (1974) 106-123

[6] J. M. Goethals, J. J. Seidel, Orthogonal matrices with zero diagonal, Canad. J. Math. 19 (1967), 1001-1010

[7] G. Greaves, J. H. Koolen, A. Munemasa, F. Szöllösi, Equiangular lines in Euclidean spaces, arXiv:1403.2155

[8] P. J. Kelly, A congruence theorem for trees, Pacific J. Math. 7 (1957) 961-968

[9] J. H. van Lint, J. J. Seidel, Equilateral point sets in elliptic geometry, Indag. Math. 28 (1966) 335-348

[10] C. L. Mallows, N. J. A. Sloane, Two-graphs, switching classes and Euler graphs are equal in number, SIAM J. Appl. Math. 28 (1975) 876-880

[11] R. E. A. C. Paley, On orthogonal matrices, J. Math. Phys. 12 (1933) 311-320

[12] S. Ramachandran, Graph reconstruction - some new developments, AKCE Int. J. Graphs Comb. 1 (2004) 51-61

[13] R. C. Read, On the number of self-complementary graphs and digraphs, J. London Math. Soc. 63 (1963) 99-104

[14] H. Sachs, Über selfstkomplementare Graphen, Publ. Math. Debrecen 9 (1962) 270-288

[15] T. Spence's homepage: http://www.maths.gla.ac.uk/ es/

[16] R. P. Stanley, Reconstruction from vertex-switching, J. Combin. Theory Ser. B 38 (1985) $132-138$ 